\title{
SISTEMA DE MEDICIÓN DE LA CALIDAD DE LA EDUCACIÓN FÍSICA EN CHILE Y SU INFLUENCIA EN LA REALIDAD ESCOLAR
}

\author{
SISTEMA DE MEDIÇÃO DA QUALIDADE DA EDUCAÇÃO FÍSICA NO CHILE \\ E SUA INFLUENNCIA NA REALIDADE ESCOLAR
}

\section{A SYSTEM FOR MEASURING THE QUALITY OF PHYSICAL EDUCATION IN CHILE AND ITS INFLUENCE ON SCHOOL EXPERIENCE}

\author{
Fernando Javier Rodriguez*, Daniel Alexis Estrada Coz*, \\ Tomás Quezada Durán*, Álvaro Andrés Tapia Guajardo*, \\ Carlos Humberto Valderrama Alvarado*, Alberto Moreno Doña*
}

\begin{abstract}
Palabras clave SIMCE.

Acondicionamiento Físico humano. Gestión de calidad.

Resumen: El actual SIMCE-EF (Sistema de Medición de la Calidad de la Educación Física), evalúa componentes de la condición física, que poco tienen que ver con los objetivos de la disciplina. El objetivo es determinar la influencia percibida por los docentes a sus prácticas educativas y en el entorno escolar, atribuida a la aplicación de la prueba SIMCE-EF. Se encuestan y entrevistan a 17 profesores de EF, pertenecientes al 100\% de los establecimientos de la Comuna de Viña del Mar, Chile, que se les aplicó el SIMCE-EF en los años, 2010, 2011 y 2012. Según los docentes, la prueba no tiene impacto en el ámbito escolar, lo que podría mantener los resultados en las aplicaciones futuras, mientras se evalúe solo la condición física y en un formato piloto.
\end{abstract}

Palavras-chave SIMCE. Condicionamento físico humano. Gestão de qualidade.

Keywords:

SIMCE.

Physical conditioning, human Quality management.

Resumo: O atual SIMCE-EF (Sistema de Medição da Qualidade da Educação Física) avalia componentes da condição física que pouco têm a ver com os objetivos da disciplina de Educação Física. $O$ objetivo deste trabalho é determinar a influência atribuída à implementação do teste SIMCE-EF pelos professores nas suas práticas educativas e no ambiente escolar. Foram entrevistados 17 professores de Educação Física, pertencentes a 100\% dos estabelecimentos do município de Viña del Mar (Chile), que Ihes aplicou o SIMCE-EF nos anos de 2010, 2011 e 2012. Segundo os docentes, os resultados encontrados indicam que SIMCE-EF não apresentam impacto no âmbito escolar, o que poderia assegurar resultados em aplicações futuras, sempre que se avalie somente a condição física em formato de estudo piloto.

Abstract: The current SIMCE-EF (Physical Education Quality Measurement System) measures elements of physical fitness that have little to do with the discipline' learning goals. The aim of this study is to determine how SIMCE-PE's influence is perceived by teachers on their own teaching practices and the school environment. Seventeen PE teachers were interviewed - a hundred percent of the public system personnel in Viña del Mar, where the exams were applied in 2010, 2011, and 2012. According to the teachers, the exam has no impact on the school and such results can remain in the future since SIMCE-PE only measures physical fitness and in a pilot format.
*Pontificia Universidad Católica de Valparaíso. Valparaíso, Chile. E-mail: fernando.rodriguez@ucv.cl

Recebido em: 19-08-2014

Aprovado em: 28-02-2015

(c) (1) (8) Licence 


\section{INTRODUCCIÓN}

La educación es un proceso de continua "re-invención", que busca permanentemente el aprendizaje de contenidos, habilidades y competencias disciplinares.

La evaluación de la calidad de la educación, se ha pretendido, aplicando diversos modelos de evaluación en los procesos educativos. En Latinoamérica, según el Observatorio Chileno de Políticas Educativas (OPECH), México y Chile han sido de los pocos países que han podido mantener en el tiempo un modelo de evaluación escolar (LABORATÓRIO LATINOAMERICANO DE LA CALIDAD DE LA EDUCACIÓN, 2008). Algunas de las más conocidas, son la prueba Third International Mathematics and Science Study (TIMSS), que consiste en una propuesta de evaluación internacional del aprendizaje escolar en matemáticas y ciencias, realizado por la International Association for the Evaluation of Educational Achievement (IEA) desde los años noventa (ACEVEDO, 2009), que se ha integrado en Brasil y Argentina (ARREGUI, 2006). La Organization for Economic Cooperation and Development (OECD), impulsa una prueba para evaluar el resultado de los sistemas educativos, que pretende determinar los aprendizajes para la vida de los estudiantes, denominada Programme for International Student Assessment (PISA), que se centra en la alfabetización lectora, matemática y científica, especialmente para los países pertenecientes a ésta organización internacional (PISA, 2012).

Chile optó por un sistema de evaluación basado en exámenes nacionales Sistema de Medición de la Calidad de la Educación Física (SIMCE), con una intencionalidad evaluativa de mejora continua pero que, pareciera haberse convertido en un sistema cuyo propósito es detectar las escuelas con bajo rendimiento académico y estigmatizarlas (MORENO; MEDINA, 2012).

En este contexto, la Agencia de Calidad de la Educación (AGENCIA DE LA CALIDAD DE LA EDUCACIÓN, 2013), acepta una propuesta hecha por especialistas para que durante el año 2010 en que se anuncia públicamente la aplicación de la nueva prueba, se implemente la primera evaluación SIMCE de Educación Física (EF), a una muestra representativa de estudiantes de $8^{\circ}$ básico de Chile. Los gobiernos hasta la actualidad, con una mirada tecnocrática de la EF y entendiéndola solo desde un punto de vista motriz, donde el ejercicio físico como objetivo toma mucha importancia, consideran necesario implementar una forma de evaluación de los componentes físicos que afectan a la salud de los escolares, para así comprobar que las prácticas docentes y temáticas concernientes a la asignatura, sean las adecuadas para ese fin. Esta evaluación contempló la aplicación de diversas pruebas físicas como la de resistencia abdominal, salto longitudinal a pies juntos, el test Navette, entre otros para medir aquellos objetivos y contenidos del currículo de EF referidos a la condición física de los estudiantes. La evaluación de la condición física tomó parte central del SIMCE de $E F$, señalando que permite obtener un diagnóstico de la condición física de los estudiantes de $8^{\circ}$ básico, el cual, junto con el informe, "[...] contribuiría a que los docentes y directivos pudieran diagnosticar a los estudiantes de sus establecimientos, y a partir de sus resultados, se reflexionara acerca de sus prácticas pedagógicas" (AGENCIA DE LA CALIDAD DE LA EDUCACIÓN, 2013, p.7).

Sin duda para los profesionales de la EF, éste ámbito evaluado deja fuera a gran parte de los competencias que le corresponden a la disciplina. Aun así a los colegios les interesa poder obtener buenos resultados en la prueba. 
La definición de Educación Física adoptada por varias escuelas formadoras de profesores de EF en Chile, se desarrolla a través del concepto de motricidad humana impulsado por Manuel Sergio (TRIGO, 2006), la cual es concebida como la forma de expresión del ser humano, como un acto intencionado y consiente, que además de las características físicas, incluye factores subjetivos, que son parte de la complejidad humana (EISENBERG, 2007), considerando un ser humano multidimensional (GONZÁLEZ; GONZÁLEZ, 2010).

Con mayor profundidad, Gómez (2008), define que la educación física es una disciplina pedagógica que debería ocuparse de crear las condiciones para el desarrollo de la corporeidad y la motricidad, dando respuesta a las necesidades del sujeto que aprende, en un contexto determinado y en una sociedad cambiante con conflictos de clase y culturas diferentes.

Pero la idea y conceptualización que se tiene de la EF es diferente a la planteada anteriormente y podría explicarse en el origen que tiene la disciplina en algunos países de América, entre ellos en Chile, a través de una fuerte influencia europea, sobre todo Alemana con la gimnasia de Friedrich Jahn, que pugna por muchos años con la gimnasia Sueca educativa promovida por Joaquín Cabezas (CORNEJO; MATUS, 2013). La gran influencia de la gimnasia Alemana con el uso de implementos y con cierta ludicidad (LANGLADE; REY, 1970), marcó fuertemente el inconsciente colectivo de nuestra sociedad. Un ejemplo semántico de ello es que las personas en Chile, aún siguen llamando a la clase de EF, como la clase de gimnasia.

Con esta mirada mono-focal, la medición de la "calidad" de la EF, está por ahora distante de ser lograda. Una revisión general hecha en relación al SIMCE (ORTIZ, 2012), plantea que el sistema mide solo una parte de los aprendizajes considerados fundamentales, lo que tendría como efecto no esperado, una reducción de la noción de calidad educativa.

En Estados Unidos el Centro de Control y Prevención de Enfermedades (CDC en inglés), crea en 2006 el Physical Education Curriculum Analysis Tool (PECAT), que señala que la determinación de la calidad de la EF debe considerar: el currículo, las políticas, el medio ambiente, la calidad de la instrucción y la evaluación de los estudiantes (CENTER FOR DISEASE CONTROL AND PREVENTION, 2006), es decir elementos que no están implícitos en la actual prueba SIMCE-EF.

Este sistema estandarizado de evaluación que se usa actualmente en la EF chilena, condiciona y modifica las prácticas educativas, pues exigen una respuesta determinada del profesorado para no ser "mal evaluados". Si a esto se le suma que el SIMCE no es coherente con el currículo chileno para la EF (MORENO; RIVERA; TRIGUEROS, 2014), entonces agregamos el problema de que los profesores se debaten entre las indicaciones curriculares y el sistema de evaluación.

Tomando en cuenta los antecedentes mencionados, la presente investigación busca determinar la "Influencia Percibida" por los docentes del área de EF, la influencia que tiene la prueba en sus prácticas educativas y en el entorno escolar de sus respectivos establecimientos, atribuida a los efectos producidos por la aplicación del SIMCE-EF. 


\section{METODOLOGÍA}

\subsection{Participantes}

La investigación corresponde a un estudio trasversal, descriptivo, con una análisis cuali-cuantitativo y con una muestra intencionada de profesores.

El grupo seleccionado para el estudio, corresponde a una muestra intencionada de 17 profesores del Subsector de Educación Física, perteneciente a la totalidad de los establecimientos de la Comuna de Viña del Mar, Chile, a quienes se les aplicó el SIMCE-EF en el año, 2010, 2011 y 2012 y que actualmente se desempeñan en labores docentes en los mismos establecimientos. Se entrevistó a docentes de establecimientos de administración Municipal, Particulares con subvención del estado y Particulares 0 privados, que corresponde al $100 \%$ de los colegios de Viña del Mar en los que se aplicó el SIMCE-EF como prueba piloto. Los docentes estaban en un rango etario de entre 24 a 58 años de edad, de los cuales siete profesores son hombres y 10 mujeres, además 14 de ellos poseen grado académico de Licenciado en Educación y 3 el grado académico de Magíster.

\subsection{Instrumentos}

Se elaboró un cuestionario de 24 preguntas cerradas, que incluyen cinco ámbitos que se consideran relevantes para el estudio. Estos ámbitos se obtienen de Trujillo y Fernández (2003), quienes plantean ocho dimensiones de calidad de la Educación Física y Deportiva Escolar: a) Valores y actitudes; b) Hábitos físico deportivos en función de una vida sana y saludable; c) Logros motrices; d) Logros cognitivos; e) Logros socio-afectivos; f) Actividades físico deportivas orientadas por profesionales de la educación física; g) Espacios adecuados para ejecución de actividades sistemáticas (infraestructura); h) Recursos didácticos (Implementación). A partir de éstos elementos se orientan las preguntas para la entrevista y se construye el cuestionario que además incluye otros elementos que pueden verse influenciados por la aplicación de la prueba SIMCE-EF.

En éste cuestionario se consulta a los docentes respecto de la pertinencia que tiene la prueba en el ámbito de la educación física y su relación con los objetivos de la disciplina y en segundo lugar la opinión de los docentes de acuerdo a la influencia que tiene la prueba en el tiempo de dedicación, la mejora de los recursos, de la actitud de los estudiantes de la clase. Elementos susceptibles a la aplicación del SIMCE-EF. De éstos ámbitos se realizan tres preguntas que corresponden a los Objetivos de la Aplicación del SIMCE-EF; cuatro preguntas sobre el Tiempo Destinado a la mejora de la prueba; sete preguntas a los Recursos y Materiales y Didácticos; seis preguntas a las Actitudes de los Estudiantes en las Clases de Educación Física; y cuatro preguntas a la Clase de Educación Física, todas relacionadas con la influencia percibida al aplicarse la prueba SIMCE-EF en su establecimiento.

Una vez completado el cuestionario, se aplica una entrevista semiestructurada de seis preguntas usando de base los cinco ámbitos antes mencionados que abarca el estudio, 
para así poder indagar con mayor profundidad en los aspectos que el cuestionario no nos permite develar.

Antes de completar los instrumentos, se rellena un consentimiento informado, el cual contiene un resumen del estudio, explicando que la información sólo será usada con fines investigativos (utilizando seudónimos para su análisis y entrega de resultados) y que en cualquier momento el profesor puede hacer preguntas referidas al estudio y así mismo retirarse del proceso de aplicación si lo estima pertinente, sin que esto lo perjudique de forma alguna. Posteriormente se aplica el cuestionario y se realiza la entrevista. Todas las aplicaciones se llevaron a cabo en los establecimientos educacionales en los que los profesionales desarrollan sus actividades docentes.

La validación de los instrumentos comprendió cuatro etapas:

1. Revisión de los Instrumentos por parte de los expertos; se solicitó a tres expertos en el área de la Educación Física (Doctorados), que revisaran las preguntas contenidas en ambos Instrumentos y que entregaran apreciaciones con respecto a la redacción, pertinencia, contenido y estructura de estos. Las principales apreciaciones fueron respecto al contenido de algunas preguntas y a la redacción de las mismas;

2. Corrección respecto a las apreciaciones de los expertos; las apreciaciones fueron recibidas y se realizaron las modificaciones pertinentes;

3. Aplicación piloto de los instrumentos; para la aplicación piloto de los instrumentos, se solicitó la participación de tres profesores de un colegio particular subvencionado de Viña del Mar. El tiempo ocupado por los docentes para responder el cuestionario fue de 7 minutos en promedio, durante la aplicación surgieron consultas referidas al contenido y la claridad de las preguntas. Por lo cual, se procedió a una nueva revisión;

4. Corrección de los instrumentos respecto a la aplicación piloto; se realizan las correcciones se acuerdo a la aplicación piloto y se finaliza el instrumento que se titula, Influencia del SIMCE de Educación Física en las Prácticas Educativas y el Entorno Escolar, Desde la Experiencia de los Profesores del Área.

\subsection{Procedimientos para el Análisis de los datos}

Para el cuestionario de preguntas cerradas, se utilizó una metodología cuantitativa para determinar resultados en porcentaje por cada pregunta. Esto se ordena en planillas de cálculo de Microsoft Excel 2010.

Respecto de las entrevistas, el primer paso para obtener los resultados, consiste en transcribirlas desde las grabaciones recopiladas durante su aplicación. Posteriormente se realiza un análisis de contenido para cada una de las respuestas por medio de la categorización, la cual posibilita clasificar conceptualmente los incidentes que son aplicables a una misma temática, puesto que una categoría contiene un significado o diferentes tipos de significados, como señala Osses, Sánchez, Ibáñez (2006), "[...] situaciones o contextos, actividades 0 acontecimientos, relaciones entre personas, comportamientos, opiniones, sentimientos, perspectivas sobre un problema, métodos, estrategias y procesos". Lo anterior, se realiza por medio de una Codificación Selectiva de conceptos (una o más palabras) que 
pueden repetir y crear un patrón común de respuestas, la cual permite reducir el conjunto inicial de categorías a partir del análisis intensivo de las relaciones entre la categoría central y el resto.

El proceso antes descrito, se lleva a cabo para codificar cada una de las preguntas de cada docente y realizar su posterior análisis.

\section{RESULTADOS}

\subsection{Resultados cuantitativos}

Los resultados de la encuesta se muestran en la tabla 1, donde se encuentran las respuestas separados en las cinco categorías de preguntas realizadas a los docentes.

A continuación se describen brevemente los resultados más importantes.

Más del $50 \%$ de las respuestas señalan que la prueba cumple medianamente los objetivos de diagnosticar la condición física y de reflexionar sobre las prácticas pedagógicas. Casi el 50\% de los docentes señala que la prueba no cumple con el objetivo de promover la actividad física y un estilo de vida saludable.

Respecto del tiempo destinado para la clase de EF a la mejora de los resultados del SIMCEEF, el $94,1 \%$, señala que se mantuvo.

En general se mantiene la dedicación temporal en los talleres extra-programáticos, se hace similar difusión de buenos hábitos de vida saludable y la participación de los estudiantes solo aumenta en un $11,8 \%$ según los docentes.

Tampoco señalan un aumento significativo del material didáctico y de la mejora de infraestructura en torno a la prueba. Excepto un $29,4 \%$ de las respuestas que indican el aumento de material pequeño de bajo costo, como balones, cuerdas, aros, etc.

De acuerdo a las actitudes de los estudiantes, las respuestas son más heterogéneas, pero se aprecia un mantenimiento de las actitudes hacia la clase de EF en torno a la prueba SIMCE, pero se destacan las respuestas en torno a que mejoran la empatía, superación y colaboración entre los estudiantes.

Finalmente los docentes responden que se mantiene la enseñanza para el aprendizaje en las clases, las responsabilidades profesionales que los docentes asumen con sus estudiantes y aumenta la preparación para la enseñanza y la creación de un ambiente propicio para el aprendizaje. 
Tabla 1 - Porcentaje de respuestas de los 17 docentes encuestados, distribuidos por ítem y preguntas.

\begin{tabular}{|c|c|c|c|c|}
\hline \multirow{4}{*}{ 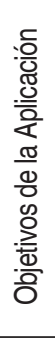 } & & $\begin{array}{l}\text { No se Cumple el } \\
\text { Objetivo }\end{array}$ & $\begin{array}{l}\text { Se Cumple } \\
\text { Medianamente el } \\
\text { Objetivo }\end{array}$ & $\begin{array}{l}\text { Se Cumple } \\
\text { Completamente } \\
\text { el Objetivo }\end{array}$ \\
\hline & Diagnosticar la Condición Física de los Estudiantes & $5,9 \%$ & $58,8 \%$ & $35,3 \%$ \\
\hline & Reflexionar acerca de las Practicas Pedagógicas utilizadas & $35,3 \%$ & $52,9 \%$ & $11,8 \%$ \\
\hline & $\begin{array}{l}\text { Elaborar Planes de Acción destinados a promover la } \\
\text { Actividad Física y un Estilo de Vida Saludable de los } \\
\text { estudiantes }\end{array}$ & $47,1 \%$ & $29,4 \%$ & $23,5 \%$ \\
\hline \multirow{5}{*}{ 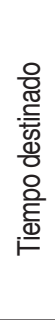 } & & Disminuyo & Se Mantuvo & Aumento \\
\hline & La clase de Educación Física & $0,0 \%$ & $94,1 \%$ & $5,9 \%$ \\
\hline & Talleres extra-programáticos & $17,6 \%$ & $70,6 \%$ & $11,8 \%$ \\
\hline & $\begin{array}{l}\text { La difusión de hábitos de vida saludable (charlas, folletos, } \\
\text { actividades promocionales, entre otras). }\end{array}$ & $5,9 \%$ & $76,5 \%$ & $17,6 \%$ \\
\hline & $\begin{array}{l}\text { La práctica de actividad física de los estudiantes en las } \\
\text { clases de EFI. }\end{array}$ & $5,9 \%$ & $82,4 \%$ & $11,8 \%$ \\
\hline \multirow{8}{*}{ 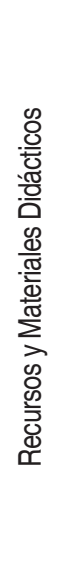 } & & Disminuyo & Se Mantuvo & Aumento \\
\hline & $\begin{array}{l}\text { Instalaciones Deportivas: Pista Atlética, Cancha, Piscina, } \\
\text { Camarines. }\end{array}$ & $0,0 \%$ & $94 \%, 1$ & $5,9 \%$ \\
\hline & $\begin{array}{l}\text { Material Deportivo: balones, petos, plintos, aros, entre } \\
\text { otros. }\end{array}$ & $0,0 \%$ & $70,6 \%$ & $29,4 \%$ \\
\hline & Equipamiento del Alumnado: Vestimenta e higiene. & $5,9 \%$ & $94,1 \%$ & $0,0 \%$ \\
\hline & $\begin{array}{l}\text { Material de Soporte al profesorado: para recopilar y/o } \\
\text { entregar información. }\end{array}$ & $5,9 \%$ & $76,5 \%$ & $17,6 \%$ \\
\hline & Material Impreso: libros, guías, fichas por ejemplo. & $5,9 \%$ & $82,4 \%$ & $11,8 \%$ \\
\hline & $\begin{array}{l}\text { Material Audiovisual e Informático: proyector, equipo de } \\
\text { música entre otros. }\end{array}$ & $5,9 \%$ & $94,1 \%$ & $0,0 \%$ \\
\hline & $\begin{array}{l}\text { Material Complementario: que son del área de EFI pelo } \\
\text { pueden ser utilizados como salones de eventos. }\end{array}$ & $5,9 \%$ & $94,1 \%$ & $0,0 \%$ \\
\hline \multirow{7}{*}{ 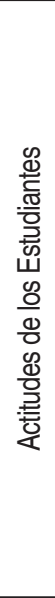 } & & Necesita Mejorar & $\begin{array}{l}\text { Se Mantuvo } \\
\text { lgual }\end{array}$ & Mejoró \\
\hline & Empatía: Escucha, comparte y apoya el esfuerzo de otros. & $17,6 \%$ & $41,2 \%$ & $41,2 \%$ \\
\hline & Iniciativa: Busca y sugiere soluciones a problemas. & $17,6 \%$ & $52,9 \%$ & $29,4 \%$ \\
\hline & $\begin{array}{l}\text { Cuidado de Materiales e Infraestructura: Utiliza de forma } \\
\text { cuidadosa los materiales e infraestructura. }\end{array}$ & $29,4 \%$ & $58,8 \%$ & $11,8 \%$ \\
\hline & $\begin{array}{l}\text { Superación Perseverancia y Esfuerzo: Demuestra } \\
\text { disposición al esfuerzo personal, superación y } \\
\text { perseverancia. }\end{array}$ & $29,4 \%$ & $23,5 \%$ & $47,1 \%$ \\
\hline & $\begin{array}{l}\text { Respecto: Respeta las variadas características físicas de } \\
\text { las personas, como altura, peso, color de piel, pelo, entre } \\
\text { otras. Respeta las reglas. }\end{array}$ & $17,6 \%$ & $47,1 \%$ & $35,3 \%$ \\
\hline & $\begin{array}{l}\text { Colaboración: Proporciona ideas cuando participa en } \\
\text { grupo y/o discusiones en clase. }\end{array}$ & $17,6 \%$ & $41,2 \%$ & $41,2 \%$ \\
\hline \multirow{5}{*}{ 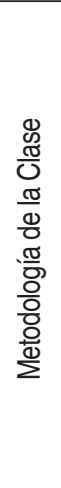 } & & Disminuyo & Se Mantuvo & Aumento \\
\hline & $\begin{array}{l}\text { Preparación de la Enseñanza, en relación a la: } \\
\text { Organización de los Objetivos y Contenidos a desarrollar. }\end{array}$ & $0,0 \%$ & $41,2 \%$ & $58,8 \%$ \\
\hline & $\begin{array}{l}\text { Creación de un ambiente propicio para el aprendizaje, } \\
\text { considerando: organización, normas, estructura de la } \\
\text { clase, entre otras. }\end{array}$ & $0,0 \%$ & $52,9 \%$ & $47,1 \%$ \\
\hline & $\begin{array}{l}\text { Enseñanza para el aprendizaje de todos, en relación al: } \\
\text { tiempo, objetivos, evaluaciones, estrategias, entre otras. }\end{array}$ & $0,0 \%$ & $82,4 \%$ & $17,6 \%$ \\
\hline & $\begin{array}{l}\text { Responsabilidades profesionales: reflexión de su } \\
\text { quehacer, orientación a estudiantes, manejo de información } \\
\text { actualizada, relación con sus pares, estudiantes, padres y } \\
\text { apoderados. }\end{array}$ & $0,0 \%$ & $64,7 \%$ & $35,3 \%$ \\
\hline
\end{tabular}




\subsection{Resultados cualitativos}

Para analizar las entrevistas, se codifican las palabras comunes para cada respuesta y se agrupan los conceptos centrales de cada una de las 17 entrevistas realizadas. Por cada pregunta se resume la respuesta de acuerdo a la mayor cantidad de respuestas similares por pregunta. Las respuestas a las preguntas de la entrevista se presentan a continuación, considerando la tendencia de las respuestas presentadas, donde en algunos casos se componen de dos partes.

Los docentes consideran que el SIMCE-EFI no está creado acorde al desarrollo motor de los estudiantes y proponen un enfoque más integral, orientando la evaluación a ámbitos motrices como: Habilidades Motoras Básicas (HMB), Habilidades Motoras Básicas Combinadas (HMBC), coordinación, juegos pre-deportivos entre otros. Una profesora de un colegio municipal plantea que, "[...] la evaluación la haría a través de un circuito con habilidades combinadas para ver tipos de coordinación y más, pero la verdad es que no encuentro ninguna relación en la prueba actual" (Paulina). Además de proponer el desarrollo de la evaluación de manera longitudinal y de evaluar el proceso de los estudiantes y no como un suceso aislado.

Al respecto otro docente municipal señala:

[...] creo que eso se mide mediante un seguimiento y esta es una medición en un tiempo específico y un espacio determinado, entonces creo que tampoco tiene relación, porque acá por ejemplo, hay un progreso de los chiquillos que no lo evalúa el SIMCE, se puede solamente a través de un seguimiento. (Carlos).

Considerando también el contexto educativo, como el nivel cultural, socioeconómico, infraestructura, horas de clases, entre otros. Los docentes manifiestan que la evaluación no tiene coherencia entre los elementos evaluados, con la labor docente.

Un docente de colegio privado explica que: "No podemos medir a los alumnos de acuerdo a las capacidades físicas que puedan obtener, de acuerdo a los test que plantean, porque no apuntan a los objetivos planteados; o sea no tienen nada que ver con la labor pedagógica [...]". (Jorge).

Otros profesores manifiestan que la prueba SIMCE-EF, evidencia la condición de salud en la que están sus estudiantes. En planteamiento de los docentes entrevistados declaran que el SIMCE-EF "no influye" de manera alguna en el tiempo dedicado, ya sea de clases y/o talleres debido a que no la consideran como una prueba importante. Por otra parte, mencionan que no hay interés institucional de aumentar las horas y/o la preparación para el SIMCE-EF ya que, el aumento o implementación de talleres está condicionado a factores externos al profesor y al establecimiento, siendo estos dependientes en ocasiones de la Corporación Municipal de Viña del Mar.

Al respecto un docente señala: "No ha aumentado nada, todo está exactamente igual, hay dos talleres extra programáticos [...] no hay nada más, porque como somos colegio municipal, es la Casa del Deporte de la Municipalidad quien coordina todas las actividades extra programáticas". (Patricia).

En las respuestas se expone que los recursos y materiales didácticos se mantienen igual y no hay inversiones importantes, ya que se contaban con materiales para desarrollar 
la batería de tests, como colchonetas, pesa, huincha para la altura, entre otros materiales básicos.

Los docentes manifiestan que los materiales disponibles han sido gestionados por razones distintas a la mejora del SIMCE-EF. Se encuentran en desarrollo proyectos de implementación de materiales, pero estos no están destinados al desarrollo de esta prueba. Esta respuesta se repite en 14 de los 17 profesores entrevistados.

Un ejemplo de ello es lo que plantea un profesor de un colegio subvencionado por el estado, "[... ]ninguna, nada, no ha habido ningún tipo de ayuda específicamente para eso... es un tema del mismo colegio, pero así específico para SIMCE, nada". (Carolina).

Indican además que son ellos mismos como profesores quienes en muchos casos gestionan la implementación para la aplicación de la evaluación, confeccionando huinchas, flexómetros artesanales, consiguiendo amplificación para el test de Navette, entre otros.

Otro componente importante es la actitud y/o el comportamiento de los estudiantes, el cual señalan que no se ha visto afectado, ya que los estudiantes tienen conocimiento de que la evaluación no involucra una calificación o identificación diferenciada en los resultados a diferencia de las demás prueba SIMCE de otras áreas, por lo tanto reconocen que no hay un incentivo inmediato de por medio, como lo es una calificación. Por otra parte, declaran que son ellos como docentes quienes se ven más afectados por el SIMCE-EF, ya que muchas veces reciben presión de la institución para obtener un buen resultado de algo que ellos no están de acuerdo.

En segundo lugar los docentes expresan que la prueba resulta ser algo novedoso para los estudiantes, por lo cual se muestran interesados y con muy buena disposición y actitud positiva hacia la evaluación en general. Conforme a ello, se esfuerzan, perseveran y ayudan entre sí y en general los hombres presentan mayor disposición al trabajo en clases que las mujeres.

Una respuesta común es que los estudiantes al tener conocimiento de la aplicación del SIMCE-EF, las clases sistemáticas se adaptan totalmente, orientado las unidades para crear y desarrollar planes de entrenamiento para mejorar las capacidades de los estudiantes que serán evaluadas, incluso quitándoles tiempo a otras unidades o simplemente dejándolas de lado, con la finalidad de preparar mejor a los estudiantes para el SIMCE-EF en las semanas previas a la aplicación de la prueba, llegando a solicitar horas a otras asignaturas.

Otra opinión es que las unidades se mantienen según lo planificado antes de saber que se les aplicaría el SIMCE-EF, y al no ser una evaluación obligatoria o con calificación particular, las clases se mantienen sin alteraciones metodológicas y/o didácticas, ya que no se encuentran condicionados aún por el SIMCE-EF. Por otro lado, al implementar talleres para trabajar la batería de test de la evaluación de forma específica, las clases sistemáticas no han sufrido alteraciones y que el desarrollar la condición física de forma transversal en las unidades, permite no dedicar tiempo específico a ellas.

\section{DISCUSIÓN DE LOS RESULTADOS}

El principal objetivo de la investigación, es poder dar cuenta de la influencia que tiene la aplicación de la prueba SIMCE-EF en los docentes y en las clases. En las entrevistas los 
docentes señalan la necesidad de que la prueba pueda evaluar desde un punto de vista más global, otros componentes como las habilidades motoras, la coordinación u otras habilidades. Es importante reconocer que la clase de EF tiene distintas unidades que los docentes van tomando a sugerencia de los planes y programas del Gobierno, como por ejemplo habilidades para deportes colectivos, habilidades de manipulación, danzas tradicionales, entre otras, y que hacen distanciar la realidad práctica que se vive en las escuelas con la evaluación SIMCE. Para Diniz y Franco (2009, p.241): "[...] la evaluación refleja la forma en que el profesor piensa su concepción del mundo, su ética, su forma de ver a los alumnos y sus conocimientos sobre los procesos de enseñanza aprendizaje en la escuela y su función", de acuerdo a éste concepto, éstos docentes intenten al menos tener una idea amplia de los contenidos y habilidades que los estudiantes requieren aprender y por lo tanto lo que se debería evaluar.

Un elemento destacable es que algunos docentes manifiestan que los estudiantes se motivan para participar de las pruebas, pero en general el ánimo y motivación por la clase se mantiene. Al menos este aspecto parece no empeorar, a pesar de que la evaluación no es lo suficientemente coherente con la realidad que manifiestan los docentes.

Otra evidencia que da cuenta de esto, se encuentra contenida en la Bases Curriculares del Ministerio de Educación para $8^{\circ}$ básico, grupo en que se realiza la prueba SIMCE-EF. En estas bases se declara en los Objetivos de Aprendizajes, tres ámbitos; a) Habilidades motrices: seleccionar, combinar y aplicar con mayor dominio las habilidades motrices específicas de locomoción, manipulación y estabilidad. b) Vida activa y saludable: Desarrollar la resistencia cardiovascular, la fuerza muscular, la velocidad y la flexibilidad para alcanzar una condición física saludable y c) Responsabilidad personal y social en el deporte y la actividad física: participar y promover una variedad de actividades físicas y/o deportivas de su interés, que se desarrollan en su comunidad escolar y/o en su entorno" (MINEDUC, 2013). Respecto del segundo objetivo de aprendizaje planteado, el $47,1 \%$ de las respuestas señalan que con la aplicación de la prueba no se cumple el objetivo, es decir no es capaz de mejorar este componente.

La Ley de Directrices y Bases Curriculares de Brasil (LDB) en su artículo 24 propone un modelo de evaluación con carácter continuo y acumulativo, con prevalencia en los aspectos cualitativos por sobre los cuantitativos (MENDES; NASCIMENTO; MENDES, 2007).

Entonces, éste SIMCE no puede dar cuenta del logro de estos objetivos si está evaluando el peso, la estatura, la velocidad alcanzada en el test de Navette, la distancia en el salto longitudinal o el número de abdominales alcanzados en un minuto, sino que responde solo a una parte de los objetivos y desde un punto de vista solo cuantitativo.

Estamos, en tanto, entendiendo al SIMCE-EF como una serie de pruebas físicas, que responden más bien a una problemática de salud, lo cual le corresponde tratar desde un punto de vista epidemiológico, a otro organismo del estado. Al respecto Moreno, Trigueros y Rivera (2012, p.50) señalan que la educación física: "[...] se ha posicionado en un paradigma que no le corresponde, ocasionando que sus contenidos en intencionalidades educativas giren en torno al desarrollo de hábitos considerados positivos por la sociedad, prescindiendo de una conceptualización crítica que lo fundamente".

Sumado a lo anterior, los docentes declaran que la prueba además es disonante con el desarrollo físico de los estudiantes de $8^{\circ}$ básico, donde por ejemplo hay algunos que pueden rendir mejor en el test de resistencia cardiovascular de Navette por su mejor desarrollo cardio- 
respiratorio y muscular, que otros de sus compañeros, que podrían tener estadios de desarrollo más bajos. Esta es una de muchas razones para reflexionar sobre los componentes de dicha prueba, donde el test Navette a pesar de estar suficientemente validado (VAN MECHLEN, 1986; ESPAÑA et al., 2010; CUENCA-GARCIA et al., 2011) parece no ser el adecuado o tal intensidad de ese test se podría transformar en un factor de riesgo cardiovascular para nuestros escolares.

Respecto del tiempo dedicado a la mejora del rendimiento en la prueba, no habría un impacto importante o influencia del SIMCE en el tiempo de la clase o la inclusión de más horas para lograr mejorar la condición física, donde los profesores podrían comenzar a enseñar las materias que serán evaluadas por el test y entrenar a los estudiantes en ejercicios y rutinas de respuesta como lo indica en su respuesta una profesora: "[...] se han quitado horas de otras asignaturas para agregarlas a las de Educación Física en pos de preparar la evaluación del SIMCE de educación física, pero se hace unas semanas antes de la aplicación". (Carolina).

En el caso de Chile, se ha demostrado que la ampliación del tiempo escolar ha tenido un impacto modesto en el tiempo instruccional y en los aprendizajes (MARTINIC; VERGARA; HUEPE, 2013), lo que es coherente con éste hallazgo, pero los componentes que se evalúan requieren de un proceso de entrenamiento de las capacidades físicas involucradas, mejorando el rendimiento de esas pruebas con entrenamiento físico. Pero sin existir una mejora cuantitativa del tiempo instruccional, que podría deberse a la administración educativa, donde el gobierno define cambios, pero las municipalidades no las implementan. La mayoría de los docentes declara que no hay aumento del tiempo o mejora significativa o interés institucional para mejorar los resultados de la prueba, lo que se podría interpretar como un bajo interés por la prueba, teniendo un bajo impacto en las políticas de los establecimientos, que se demuestra en la encuesta y en las entrevistas, donde se mantienen los recursos y no hay inversiones importantes que vayan en mejora de la calidad de los procesos de enseñanza.

De alguna manera puede que las autoridades de los establecimientos comprendan la problemática y no avancen en la mejora de materiales e infraestructura hasta que no se resuelva definitivamente la aplicación obligatoria de la prueba. No obstante en la Quinta Conferencia Internacional de Ministros y Altos Funcionarios Encargados de la Educación Física y el Deporte, declaran: "[...] una inversión sostenida en educación física de calidad no es una opción de política sino un componente fundamental de la filosofía de todos los países [...]". (UNESCO, 2013, p.7), definición que las autoridades educacionales no pueden olvidar.

Los docentes perciben que sus estudiantes, en general, no mejoran ni empeoran su actitud o motivación por la presencia de la prueba, no habiendo una preocupación importante por el mayor o menor puntaje obtenido.

Afortunadamente no se entregan los resultados por establecimiento de manera pública, ya que según Cañoles (2011) esto sería perjudicial para la adherencia al ejercicio en aquellos centros en los cuáles los resultados fuesen insatisfactorios con el riesgo de estigmatizar a la población que obtiene resultados desfavorables, además correríamos el riesgo de enfocar la clase de EF a la obtención y mejora de resultados, hecho que descontextualizaría el sentido lúdico y formativo de la EF.

Los profesores no cambian sus metodologías u orientaciones de las sesiones para la mejora del rendimiento de la prueba, se cree que con lo que practican en clases es suficiente 
y no realizan grandes cambios a excepción de algunas semanas antes de la aplicación donde dedican algo más de tiempo.

La mejora de la calidad de las sesiones es importante y no puede perderse el horizonte solo con el aumento de las horas (FLINTOFF; FOSTER; WYSTAWNOHA, 2011), en éste caso tal aumento no tiene un impacto cualitativo en la disciplina, mientras se evalúe desde un punto de vista cuantitativo.

\section{CONCLUSIONES}

EI SIMCE de Educación física ha estado presente en el debate académico de la disciplina en Chile. A nivel escolar, los docentes sin tener más opciones y al ser elegidos sus colegios para que se les aplique la prueba piloto, no les queda otra opción que aceptar dicho proceso, además de manera no participativa.

No se evidencia una influencia de la prueba SIMCE-EF en el aumento de las horas para su preparación, ni mucho menos la inclusión de recursos para mejora de la calidad de las clases ni del rendimiento físico de los escolares, aunque según las reflexiones no debería realizarse, si esperamos cualificar la calidad de la enseñanza. Al respecto se evidencia una falta de coherencia entre las políticas educativas y la capacidad que tienen los sistemas de administración escolar, como las municipalidades, de aportar a dichas metas propuestas.

Los docentes opinan que no hay una relación entre los objetivos de la prueba y la manera de cómo se aplica a través de los diferentes test.

A los estudiantes por su parte, parece no afectarles el rendimiento que puedan tener en dicha prueba, pero previo a su aplicación, al parecer están más atentos y motivados.

En resumen, la prueba SIMCE-EF, según éste grupo de docentes, parece no tener un impacto en el ámbito escolar, lo que podría verse reflejado en un mantenimiento de los malos resultados en las aplicaciones futuras, mientras se mantenga en una lógica de la condición física y en un formato piloto.

\section{REFERENCIAS}

ACEVEDO, José Antonio. TIMSS Y PISA: dos proyectos internacionales de evaluación del aprendizaje escolar en ciencias. Revista Eureka sobre Enseñanza y Divulgación de las Ciencias, Cádiz, v. 2, n. 3, p.282-301, 2005.

AGENCIA DE CALIDAD DE LA EDUCACIÓN. Informe de Resultados SIMCE Educación Física 8 Básico 2012 para Docentes y Directivos. Santiago de Chile, 2012. Disponible en: < http://www.agenciaeducacion.cl/wp-content/uploads/2013/08/WEB IR 8 basico Ed Fisica 2012.pdf >. Acceso en: 10 ene. 2014.

ARREGUI, Patricia. Sobre Estándares y Evaluaciones en América Latina. Santiago: PREAL, 2006.

OSSES BUSTINGORRY, Sonia; SÁNCHEZ TAPIA, Ingrid; IBÁÑEZ MANSILLA, Flor Marina. Investigación cualitativa en educación: hacia la generación de teoría a través del proceso analítico. Estudios pedagógicos,Valdivia, v. 32, n. 1, p. 119-133, 2006 
CAÑOLES, Felipe. Sistema de medición de la calidad de la educación física en Chile: análisis crítico sobre el estado de la cuestión. EFDeportes.com, Revista Digital. Buenos Aires, v. 15, n. 154, 2011. Disponible en: <http://www. efdeportes.com/efd154/sistema-de-medicion-de-la-calidad-dela-educacion-fisica-en-chile.htm>. Acceso en: 2 sept. 2014.

\section{CENTER FOR DISEASE CONTROL AND PREVENTION. Physical Education Curriculum Analysis Tool. Atlanta, 2006.}

CORNEJO, Miguel; MATUS, Carlos. Educación física en Chile. Revista Electrónica Actividad Física y Ciencias, Maracay, v. 5, n. 1, p.1-25, 2013.

CUENCA-GARCIA, Magdalena; JIMÉNEZ-PAVÓN, David; ESPAÑA-ROMERO, Vanesa; ARTERO, Enrique; CASTRO-PIÑERO, José; ORTEGA, Francisco; RUIZ, Jonatan; CASTILLO; Manuel. Condición física relacionada con la salud y hábitos de alimentación en niños y adolescentes: propuesta de addendum al informe de salud escolar. Revista de Investigación en Educación, Vigo, v. 9, n. 2, p.35-50, 2011.

DINIZ, Josiane; FRANCO, Silvia Cristina Amaral. A avaliação na Educação Física Escolar: uma comparação entre as escolas tradicional e ciclada. Movimento, Porto Alegre, v. 15, n. 1, p. 241258, 2009.

EISENBERG, Rose (Coord.). Corporeidad, movimiento y educación física 1992-2004. México: Grupo Ideograma Editores, 2007. (La investigación educativa en México 1992-2002, v. 12).

ESPAÑA-ROMERO, Vanesa; ARTERO, Enrique; JIMÉNEZ-PAVÓN, David; CUENCA-GARCÍA, Magdalena; ORTEGA, Francisco; CASTRO-PIÑERO, José; SJOSTROM, Michael; CASTILLOGARZÓN, Manuel; RUIZ, Jonatan. Assessing health-related fitness tests in the school setting: reliability, feasibility and safety; the ALPHA Study. International Journal Sports Medicine, Stuttgart, v 31, n.7, p.490-497, 2010.

FLINTOFF, Anne; FOSTER, Rebecca; WYSTAWNOHA, Simon. Promoting and sustaining high quality physical education and school sport through school sport partnerships. European Physical Education Review, Chester, v.17, n.3, p.341-351, 2011.

GÓMEZ, Jorge. Educación Física: La educación física y su contenido. Buenos Aires: Ministerio de Educación, Ciencia y Tecnología de la Nación. Dirección Nacional de Gestión Curricular y Formación Docente Áreas Curriculares, 2008.

GONZÁLEZ-CORREA, Aída M.; GONZÁLEZ-CORREA, Clara H.. Educación Física desde la corporeidad y la motricidad. Hacia promoc. Salud, Manizales, v.15, n.2, p. 173-187, 2010.

LANGLADE, Alberto; REY DE LANGLADE, Nelly. Teoría general de la gimnasia. Buenos Aires :Stadium, 1970. p. 348.

LABORATORIO Latinoamericano de Evaluación de la Calidad de la Educación. Reflexiones en torno a la evaluación de la calidad educativa en América Latina y el Caribe. Santiago: Salesianos, 2008.

MARTINIC, Sergio; VERGARA, Claudia; HUEPE, David. The use of time and interactions in the classroom: a case study in Chile. Pro-Posições, Campinas, v. 24, n. 1, p.123-135, 2013.

MENDES, Evandra; NASCIMENTO, Juarez; MENDES, José. Metamorfoses na avaliação em Educação Física: da formação inicial à prática pedagógica escolar. Movimento, Porto Alegre, v. 13, n. 2, p. 55-76, 2007. 
MINEDUC. Bases Curriculares: $7^{\circ}$ básico a $2^{\circ}$ medio, 2013.Disponible em: curriculumenlinea. mineduc.cl/descargar.php?id_doc=201405121617150. Acceso en: 8 mayo 2014.

MORENO, Alberto; MEDINA, José Manuel. Escuela, educación física y transformación social. Estudios pedagógicos ,Valdivia, v. 38, n. esp., p.7-11, 2012.

MORENO, Alberto; RIVERA, Enrique; TRIGUEROS, Carmen. Sistema de medición de la calidad de la Educación Física Chilena: un análisis crítico. Movimento, Porto Alegre, v. 20, n. 1, p. 145167, 2014.

MORENO, Alberto; TRIGUEROS, Carmen; RIVERA, Enrique. El papel de la educación física escolar en el siglo XXI: una mirada desde el docente universitario. Movimento, Porto Alegre, v. 18, n.4 p., 33-54, 2012.

ORTIZ, Iván. En torno a la validez del Sistema de Medición de la Calidad de la Educación en Chile. Estudos pedagógicos, Valdivia, v. 38, n. 2, p. 355-373, 2012.

PISA. Assessment and Analytical Framework Mathematics, Reading, Science, Problem Solving and Financial Literacy. Paris: OECD, 2012.

TRIGO, Eugenia- Motricidad y creatividad en la escuela. Revista Motricidad y persona, Santiago, v 1, n 1, p 26-38, 2006.

TRUJILLO, Héctor; FERNANDEZ, Omar. Calidad de la Educación Física escolar: un concepto en proceso de legitimación. Revista Motricidad Humana, Valparaíso, v. 1, p. 8-10, 2003.

UNESCO. Declaración de Berlín. In: CONFERENCIA INTERNACIONAL DE MINISTROS Y ALTOS FUNCIONARIOS ENCARGADOS DE LA EDUCACIÓN FÍSICA Y EL DEPORTE,5. 2013. Disponible en: < http://unesdoc.unesco.org/images/0022/002266/226638s.pdf $>$. Acceso en: 3 jul. 2014.

VAN MECHLEN, Willem; HLOBIL, Hotol; KEMPER Han. Validation of two running tests as estimates of maximal aerobic power in children. European Journal Applied Physiologyg, Heidelberg, v. 55, n. 5, p.503-506, 1986.

\section{AGRADECIMIENTOS}

Este artículo fue realizado en el contexto y gracias al patrocinio del Proyecto de Investigación FONDECYT (Fondo de Investigación Científica y Tecnológica) № 11130408, titulado "SIMCE de Educación Física: Diseño, Aplicación y Evaluación de una nueva Propuesta" 